\title{
Deux modèles constructivistes pour le diagnostic des communications organisationnelles
}

\section{Alex Mucchielli}

\section{(2) OpenEdition}

1 Journals

Édition électronique

URL : http://journals.openedition.org/communicationorganisation/3442

DOI : 10.4000/communicationorganisation.3442

ISSN : $1775-3546$

Éditeur

Presses universitaires de Bordeaux

Édition imprimée

Date de publication : 1 octobre 2006

Pagination : $12-46$

ISSN : 1168-5549

\section{Référence électronique}

Alex Mucchielli, « Deux modèles constructivistes pour le diagnostic des communications

organisationnelles », Communication et organisation [En ligne], 30 | 2006, mis en ligne le 21 juin 2012, consulté le 19 avril 2019. URL : http://journals.openedition.org/communicationorganisation/3442 ;

DOI : 10.4000/communicationorganisation.3442

Ce document a été généré automatiquement le 19 avril 2019.

(c) Presses universitaires de Bordeaux 


\title{
Deux modèles constructivistes pour le diagnostic des communications organisationnelles
}

\author{
Alex Mucchielli
}

1 La « modélisation » n'est pas définie comme l'opération de construction de modèles mais, dans une perspective constructiviste, comme la construction d'une représentation schématisée du fonctionnement des phénomènes étudiés, représentation obtenue à partir d'une théorie ou de son modèle. La "modélisation », en tant que résultat schématisé de la recherche, joue alors, dans la perspective subjectiviste-constructionniste, le rôle de l'expérimentation dans la perspective positiviste.

2 Cet article s'attache à montrer les intérêts et les limites de deux modélisations utilisées pour le diagnostic des échanges dans les organisations : le modèle de la systémique dite «qualitative » et le modèle dit «situationnel». Ces modèles sont valorisés pour leur nouveauté et surtout pour leurs caractéristiques dites "d'ouverture». En effet, contrairement aux autres modèles, ils travaillent avec des "théories lâches " et des "macro-concepts», ce qui laisse une large marge d'invention inductive possible aux chercheurs qui les utilisent.

\section{Généralités sur la connaissance scientifique}

\section{La finalité des approches scientifiques}

Les sciences de la communication, comme toutes les sciences, visent à rendre «intelligibles» les phénomènes qui rentrent dans leur champ d'analyse. L'intelligibilité scientifique est essentiellement faite de sens partagé. Or, le sens naît toujours d'une mise en relation avec quelque chose. Le sens est en effet toujours sens de quelque chose dans ou par rapport à quelque chose. Le sens (et donc l'intelligibilité scientifique), naît de la confrontation de ce que nous appelons la "réalité» à un certain nombre de références servant de projet de décodage. Les principaux référents constitutifs de cette 
appréhension de la « réalité» pour la transformer en « représentation scientifique » sont les théories et les modèles qui découlent de ces théories.

\section{Modèles et modélisations}

4 Le terme de modèle, nous dit Sinaceur (1999, p. 651), présente une grande variété d'acceptions dans les sciences. Un modèle, ce peut être la simplification d'une théorie, la reproduction analogique d'une réalité concrète, la formalisation logique d'un ensemble de propriétés, la mise en équation d'un ensemble d'observations et de mesures, un échantillon concret d'un fonctionnement particulier, ou encore une réalisation matérielle du type « maquette ».

5 L'activité de «modélisation », quant à elle, fonctionne dans le sens inductif comme dans le sens déductif-applicatif. Dans le sens inductif, elle mène, des données empiriques à leur formalisation. Ainsi, dit-on que l'on "modélise " lorsque l'on trouve des principes ou des équations formelles dont la mise en oeuvre rend compte du phénomène étudié. On peut aussi «modéliser » en partant des mêmes données empiriques mais pour construire une réalisation physique dont le fonctionnement simule le phénomène. Ainsi on peut modéliser des phénomènes de comportements de foule soit à l'aide d'équations ou de règles logiques, soit à l'aide d'un dispositif électronique concret rassemblant de multiples « agents » électroniques programmés.

Quoi qu'il en soit, conclut Sinaceur, « un modèle fait toujours fonction de médiateur entre un champ théorique dont il est une interprétation et un champ empirique dont il est une formalisation ». Un modèle permet fondamentalement de « reproduire le résultat d'un enchaînement d'évènements" (Granger, 2003, p. 189). Il est aussi toujours une simplification, soit d'une théorie, soit des phénomènes empiriques dont il veut rendre compte. Il peut donc remplir divers rôles selon la manière dont il a été construit et les finalités de son utilisation par le chercheur: celui d'illustration, celui de support du raisonnement ou encore, celui de support à la simulation intellectuelle d'expériences.

7 Les modèles participent, comme nous venons de l'évoquer, à la constitution du sens scientifique des phénomènes. C'est parce que l'on rapporte tel phénomène à tel «modèle » qu'on en organise la compréhension de telle ou telle manière et que cette compréhension peut être partagée. Les modèles ont donc des fonctions générales qui sont des fonctions de connaissance et de partage (comme les théories). « [Le modèle, c'est] un intermédiaire à qui nous déléguons la fonction de connaissance, plus précisément de réduction de l'encore énigmatique en présence d'un champ d'études dont l'accès pour des raisons diverses, nous est difficile.» (S. Bachelard, 1979, t. 1, p. 3.)

\section{Théorie}

De façon générale, une théorie, nous dit Hempel, a pour but d'apporter une compréhension plus approfondie et plus exacte des relations mises en évidence entre des phénomènes. "Une théorie, à cet effet, interprète ces phénomènes comme les manifestations d'entités ou de processus situés à l'arrière plan, [...] ces entités et processus étant régis par des lois ou des principes théoriques caractéristiques, grâce auxquels la théorie explique alors les relations découvertes.» (Hempel, op. cit., p. 109.) 
9 Une théorie propose donc une organisation du monde des phénomènes dont elle s'occupe. La théorie électromagnétique, par exemple, nous montre comment sont structurés les champs magnétiques à partir de pôles électriques et comment, dans des champs magnétiques, se déplacent les différents corpuscules constitutifs de la matière. Elle nous présente une organisation de ce «monde magnétique» reposant sur des lois. Elle nous montre enfin comment et où regarder pour « voir » tel ou tel phénomène. Il en est de même en sciences humaines et sociales. La «théorie systémique qualitative des communications » par exemple, nous dit que chaque «segment de communication » est inséré dans un "système de communication », et que ce segment prend son sens dans le système, tout en étant lié, par des causalités circulaires aux autres communications faites par les autres acteurs... Une telle théorie contient d'ailleurs tout un ensemble de "prescriptions méthodologiques». En fait, elle ne nous laisse pas libre de nos observations. La théorie à laquelle on se réfère plus ou moins clairement, intervient donc même au niveau du recueil des données (ne serait-ce que parce qu'elle fournit des concepts que l'on met en œuvre dans les tout premiers recueils de données).

\section{Modèle théorique}

10 Un «modèle théorique" peut d'abord être considéré comme une représentation simplifiée d'une théorie. Il s'agit, le plus souvent, d'une représentation schématique (mais ce peut être une description discursive du fonctionnement canonique des phénomènes). Cette représentation ou présentation est essentiellement à but pédagogique : elle est faite pour mieux faire comprendre la théorie. La théorie a pour but de nous faire comprendre comment les phénomènes qu'elle étudie fonctionnent. Mais le modèle, de par sa simplification, marque les chercheurs qui l'intègrent et ils s'en servent alors comme guide de réflexion. En conclusion, un modèle guide donc l'observation "scientifique " puisqu'il porte, dans sa description et dans son fonctionnement, les concepts essentiels de la théorie qu'il transcrit et qu'il précise aussi le fonctionnement des phénomènes (il dit où il faut regarder et ce que sont les processus que l'on devrait voir). Il en est ainsi, par exemple, du fameux modèle "Emetteur-Récepteur» de la théorie de l'information: il indique comment fonctionne la transmission de l'information et il propose de regarder ce phénomène avec des concepts qui sont schématisés dans sa représentation graphique. Ces " concepts »: émetteur, récepteur, codage, décodage, canal de transmission, message, contenu du message, bruit, feed-back,..., orientent l'observation des phénomènes de transmission de l'information. Ces concepts, sont par ailleurs, liés entre eux dans le fonctionnement que la théorie propose (dans le modèle E-R, il y a un enchaînement canonique qui rend compte de la transmission, du décodage et de leurs avatars).

Image1Dans cette conception, un modèle théorique est donc comme une paire de lunettes déformantes. À partir du moment où l'on a intégré intellectuellement un modèle, ce modèle agit comme un mécanisme perceptif et cognitif qui transforme la "réalité» en représentation (Le Moigne). Un modèle, c'est donc, au final, un mécanisme de sélection et de recomposition qui est destiné, à rendre intelligible une réalité (à lui donner du sens en référence à une théorie qui propose une explication du fonctionnement d'un type de phénomènes). Le modèle théorique agit donc comme un processus transformateur que le chercheur met en œuvre dans son effort de construction de l'objet scientifique de sa recherche. Dès que la réalité est un tant soit peu complexe, le modèle de référence est forcément réducteur. Par ailleurs, la représentation scientifique élaborée grâce au 
modèle porte les traces des orientations du modèle de référence. Si ce «transformateur est mécanique, nos représentations scientifiques seront mécaniques ; s'il est systémique, nos représentations scientifiques seront systémiques" (Le Moigne, 1984). Le modèle théorique est donc porteur d'une certaine "interprétation» des phénomènes. Cette interprétation va nous faire voir ce qui est déjà contenu dans le "guide d'observation» que constitue ce modèle.

Fig. 1. Place et fonctionnement du modèle théorique dans le « cycle paradigmatique de la recherche "

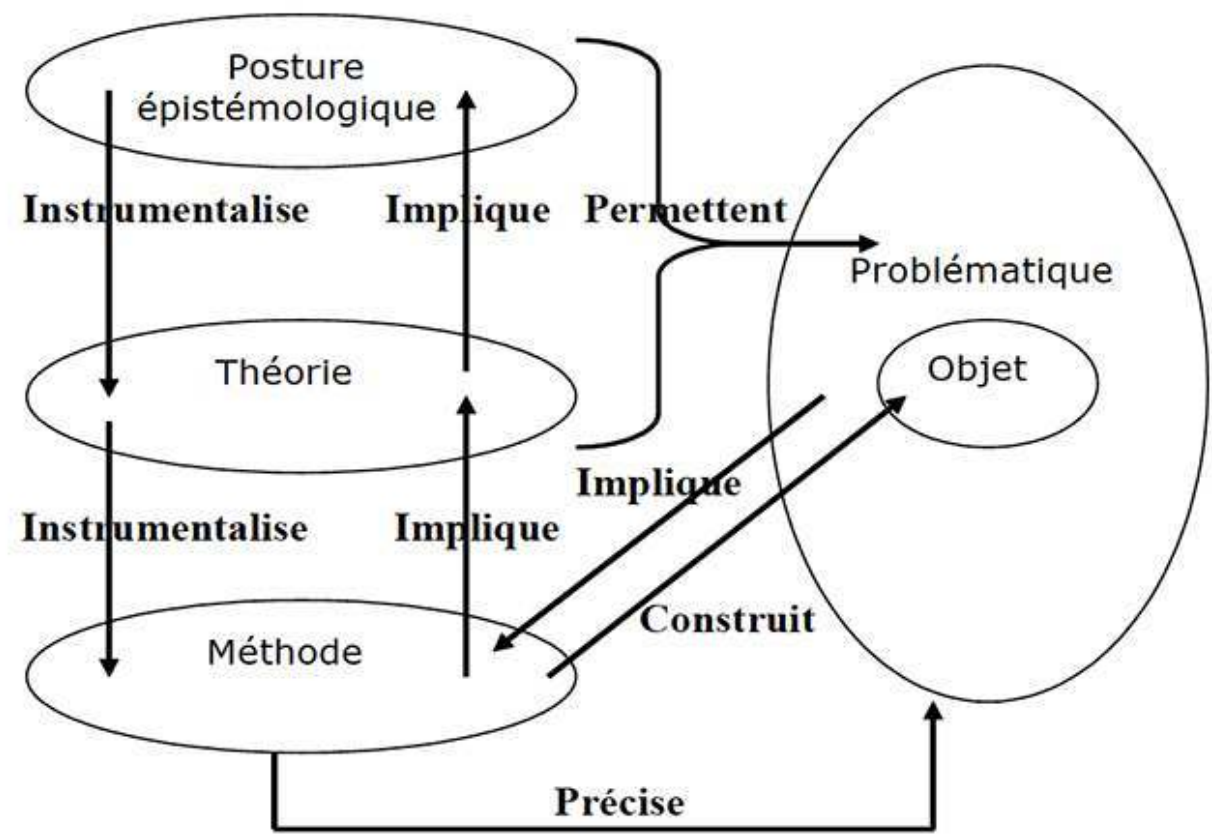

11 Un modèle théorique trouve sa place dans un ensemble qui comprend la posture épistémologique du chercheur, la théorie qu'il reflète, la problématique de recherche et l'objet de recherche, ainsi que la méthode utilisée. J'ai proposé d'appeler cet ensemble, le "cycle paradigmatique du fonctionnement de la recherche scientifique». On peut représenter ce cycle justement par une sorte de « modèle » (cf. figure 1).

\section{Ensemble paradigmatique dont dépend une recherche en sciences humaines}

\section{La " posture épistémologique »}

12 La "posture épistémologique » du chercheur correspond à ses a priori sur la science en général et sur la nature de la connaissance scientifique. Tout chercheur a des « croyances » sur la science et ses résultats. Il y a trois grandes postures épistémologiques reconnues actuellement: la posture positiviste, la posture subjectiviste et la posture constructiviste. Il faut aussi dire que les philosophes des sciences sont d'accord sur le fait qu'un chercheur, ne "peut pas ne pas » avoir de posture épistémologique. Même (et 
surtout), s'il prétend ne pas en avoir, il est porteur malgré lui d'un ensemble d'a priori qui sont le résultat de son "acculturation scientifique » (autrement dit de tout ce qu'il a appris, par immersion culturelle dans un milieu de recherche, sur la connaissance scientifique).

La posture épistémologique du chercheur "instrumentalise » (c'est-à-dire favorise en tant qu'outil), certaines formes de théories (et les modèles qui vont avec). Si l'on est positiviste, par exemple, on ne va pas travailler avec des théories et des modèles de l'émergence contextualisante du sens, mais avec des théories causalistes, donnant des explications précises du phénomène d'attribution du sens par les acteurs (codage, par exemple). Les théories que l'on est prédisposé à utiliser sont nécessairement en cohérence avec la posture épistémologique que l'on a. Ceci explique que les chercheurs « positiviste » et « réalistes » (par exemple), ne puissent pas utiliser des théories et des modèles subjectivistes ou constructivistes : ils n'arrivent pas à raisonner avec ces théories et ces modèles; ceux-ci s'opposent trop à leurs convictions profondes. De plus, si un chercheur adopte une théorie et son modèle, cette acceptation implique le point de vue épistémologique qui va avec. Les théories et leurs modèles sont donc liés à des postures épistémologiques. La théorie freudienne et ses modèles du fonctionnement du psychisme, par exemple, théorie qui est positiviste-causaliste-réaliste ne peut fonctionner dans un référentiel subjectiviste. Si un chercheur entreprend de raisonner en partie avec la théorie freudienne et en partie avec la systémique qualitative, laquelle appartient à un paradigme subjectiviste, il ne pourra pas concilier les concepts de ces théories dans les "explications" des phénomènes qu'il donnera. En fait, si l'on analyse bien les résultats fournis par ceux qui tentent ce genre d'éclectisme, on se rend compte qu'ils ne font que "traduire" des éléments d'une théorie dans l'autre (celle qu'ils connaissent le mieux). Ainsi, les freudiens se livrant à de l'analyse systémique, «ramènent » des observations, faussement étiquetées avec des concepts systémiques, à leurs interprétations canoniques, issues du modèle psychanalytique.

\section{Place et fonctionnement de l'ensemble : posture épistémologique, théorie et modèle}

L'ensemble "posture épistémologique, théorie et modèle ", permet de poser une problématique au sujet d'un "objet » scientifique à étudier. Ce phénomène est essentiel dans la recherche. On pose nécessairement des problèmes de recherche en accord avec la théorie et le modèle que l'on prend comme référence.

Par exemple, avec la théorie de l'information et le modèle Emetteur-Récepteur, on se demandera pourquoi tels ou tels services, dans une entreprise, ne se comprennent pas et se livrent une guerre larvée pour faire passer leurs idées à la direction. Mais la réponse se trouvera en fonction de ce que le modèle permet de présupposer : il y a sans doute une « incompréhension » due à une interprétation différentes des choses : de la situation, des informations stratégiques, des informations marketing, des travaux donnés par la direction,... Il y a donc une série de codages-décodages différents due aux grilles interprétatives différentes.

Quant-à la lutte pour faire passer ses idées, si on ne prend pas le modèle très prégnant de la sociologie des organisations (lutte pour le pouvoir et pour accroître ses zones d'incertitude), on prendra ce qui est donné avec la théorie de l'information : bataille pour faire partager sa «représentation » des choses par la direction et donc pour étendre à 
tous cette «représentation» (qui est le «résultat» d'une addition de messages informatifs dans la tête des uns et des autres). On étudiera alors les moyens utilisés pour faire passer ces «messages» (les canaux) et les «contenus» de ces messages (à travers des analyses de contenus). En fait, on le voit bien, les résultats ne vont faire que valider le modèle sous-jacent en le remplissant avec des éléments concrets spécifiques du terrain d'étude. Dans cet exemple, l'objet d'étude : une bataille d'information, est complètement corrélatif, c'est-à-dire dépendant, de la théorie et du modèle de référence.

\section{Place et fonctionnement de la méthode}

méthode de recherche utilisée est en relation directe avec la théorie et son modèle. Ceux-ci déterminent grandement le type des techniques de recueil et d'analyse retenues dans la méthode. A ce sujet, il est assez faux de dire qu'une technique peut être « neutre ». Par exemple, même une interview non directive fonctionne encadrée par la problématique posée, laquelle est entièrement dépendante de la théorie, de son modèle et aussi de la posture épistémologique du chercheur. Le questionnement du chercheur ne peut donc être «naïf ». Il est encapsulé dans la théorie et dans la problématique. Lorsqu'il interroge, lorsqu'il observe, ..., il n'est pas à attendre que les «faits» lui sautent à la figure et qu'ils parlent d'eux-mêmes. Il sait qu'il «va faire parler ces faits» car il recherche, in fine, à vérifier un fonctionnement qu'il a formulé comme une hypothèse implicite (celui contenu dans la théorie et le modèle).

Par ailleurs, il est indéniable que la méthode, dans le cours de sa mise en œuvre, précise sans arrêt la problématique de la recherche et construit, en continu, l'objet de la recherche.

$\mathrm{Si}$, par exemple, l'on travaille sur la «communication-participation " (qui est un des concepts proposés par la théorie systémique qualitative des communications), il est évident, que seule l'utilisation de certaines méthodes de recueil va permettre la construction du système des communications recherché. Chaque théorie ne peut que proposer des méthodes d'analyse cohérentes avec elle. On ne voit pas, par exemple, comment une "analyse de contenu » des échanges permettrait de faire une "analyse systémique qualitative» d'un ensemble de communications. La notion de « contenu » est, en effet, antinomique avec le concept de «forme signifiante des communications » que met en évidence l'analyse systémique qualitative. Une « forme signifiante » émerge d'une mise en relation d'un échange récurrent avec l'ensemble du système des communications, alors qu'un «contenu », se trouve dans le «message » lui-même, indépendamment de la «logique du système » dans laquelle il se trouve nécessairement placé. Par ailleurs, le contenu ne peut, par exemple encore, donner accès aux boucles de causalité circulaire (sur la « rupture paradigmatique» entre la notion de message et la notion d'interaction, on se rapportera, notamment, à P. Watzlawick dans son ouvrage fondateur de la notion de communication-participation, Une Logique de la communication (1972).

\section{La totalité paradigmatique}

Ainsi donc, la théorie et son modèle, compte tenu de la problématique de recherche, impliquent de prendre une méthode adaptée. L'ensemble choisi (posture, théorie-modèle et méthode), intervient en retour sur la problématique de la recherche. L'objet scientifiquement construit dépend largement de cet appareillage intellectuel. Toute 
recherche scientifique, en sciences humaines, est liée à l'ensemble: position épistémologique du chercheur/théorie modèle et méthode/ problématique et objet.Cet ensemble forme un tout indissociable où chaque élément dépend des autres et renvoie aux autres.

\section{Un modèle « systémique » pour le diagnostic des communications organisationnelles}

\section{La théorie de référence}

21 La théorie systémique qualitative (et son modèle), comme toute les théories, a une série d' a priori sur les phénomènes constatés. Dans notre cas, la théorie de référence est une «théorie lâche ». Elle s'appuie sur quelques principes d'une extrême généralité que nous allons rappeler (Mucchielli, 2004). Par ailleurs, les concepts utilisés dans la théorie sont des « macro-concepts » au sens de Morin : ce sont des catégories notionnelles larges qu'il convient toujours de préciser sur le terrain, c'est-à-dire auxquelles il faut trouver une définition concrète (ce travail de spécification du macro-concept, par l'expérimentation intellectuelle sur le terrain, étant, nous le verrons, une des conditions pour que la méthode soit dite « constructiviste »).

Rappelons que les systèmes de communication que l'on étudie en sciences humaines et sociales sont différents des systèmes formels ou mécaniques des sciences de l'ingénieur et encore plus des systèmes utilisés dans les sciences mathématiques et physiques. L'existence de ces différentes sortes de "modèles systémiques", sème d'ailleurs la confusion dans les sciences humaines (Mucchielli, 2006, pp. 11-61). Dans l'approche et l'analyse des systèmes par les sciences mathématiques ou les sciences de l'ingénieur, il n'y a jamais de possibilité de prise en compte de significations humaines des interactions, pour la bonne raison que les activités étudiées ne sont pas des échanges entre humains, mais des échanges entre entités physiques ou mathématiques ou entre machines.

Un système de communications, au sens de la systémique qualitative des communications, est un ensemble récurrent, régulier et repérable de formes d'échanges existant, dans une certaine temporalité, entre des acteurs participant d'un cadre d'action pertinent, ensemble qui entraine les acteurs dans sa dynamique propre. Les « formes des échanges " prennent, par ailleurs, des significations propres lorsqu'on les rapporte au système total des échanges. Cet ensemble peut être schématisé de telle sorte que l'on puisse parcourir, en divers circuits, les communications qui s'enchaînent les unes les autres en s'impliquant réciproquement, et, que l'on puisse accéder à une compréhension globale de cet enchaînement à travers la construction de la logique (ou problématique) de son fonctionnement.

En fait, un système de communications est un précipité d'un ensemble de situations. C'est une sorte d'idéal-type d'interactions répétitives dans leurs formes, construit par sédimentation de l'observation de nombreuses situations concrètes. Dans ces situations, on n'a relevé que certains échanges et on en a repéré des "formes" (ou catégories) d'échanges récurrents. Le relevé, orientant la définition de la situation idéal-typique finale, est finalement fait à partir d'une formulation de la logique du « jeu » joué entre les acteurs (« logique » qui est une autre forme de l'enjeu collectif des acteurs dans le jeu). 


\section{Les principes de la théorie systémique de référence}

L'approche systémique qualitative s'inscrit d'abord dans le cadre de l'approche compréhensive. Mais, à cette approche particulière des phénomènes humains de communication, s'ajoute sept principes spécifiques à ceux de l'approche compréhensive classique. Comme je l'ai dit, ces principes épistémologiques orientent donc fortement le regard et le recueil des « données ».

\section{1- Le principe systémique}

Un phénomène isolé de communication n'existe pas, il doit toujours être considéré comme étant en interaction avec d'autres phénomènes de communication.

\section{2- Le principe du niveau d'observation ou de cadrage}

Un phénomène de communication n'est compréhensible (ne prend son sens) que replacé dans un ensemble qu'il convient de délimiter. Ce cadrage définit les acteurs à prendre en compte. Par ailleurs ce cadrage n'est pas indépendant des problèmes qu'il permet d'atteindre.

\section{3- Le principe du primat du contexte systémique}

Les phénomènes de communication prennent leur sens dans le contexte formé par le système lui-même.

\section{4- Le principe de récursivité}

Chaque phénomène de communication est pris dans un jeu complexe d'implications mutuelles d'actions et de rétroactions.

\section{5- Le principe de stabilité}

Un système de communications est repérable à cause de sa stabilité. Les "données " recueillies au début de l'observation sont des " communications concrètes récurrentes ", c'est-à-dire des communications qui se répètent, ont des «formes » analogues. Par ailleurs, un système de communications a ses propres règles de fonctionnement définissant une «logique» globale de fonctionnement, laquelle constitue une force interne, propre à sa reproduction.

\section{6- Le principe d'émergence}

31 Dans un système de communications, chaque sous-système interne est à la fois autonome et contraint, organisé et organisateur, informant et informé et il convient d'en tenir compte. Cette «structure, structurée et structurante » fait émerger des phénomènes de sens. 


\section{7- Le principe de la nature de la communication prise en compte} explicitée, au final, dans la dernière modélisation, sous la forme d'une catégorie signifiante d'échange. Pour être portée sur la modélisation, la catégorie d'échange doit généraliser de nombreux contenus concrets observés et ayant la même signification dans la « logique ».

\section{Les règles de la modélisation systémique des communications}

La théorie systémique qualitative des communications est comme certaines théories : elle n'a pas de modèle schématique concret. Son «modèle » est un modèle discursif, fait d'un ensemble de "règles" assez proches des principes de la "théorie » elle-même. On retrouve la même chose, par exemple, avec la théorie de l'Ecole de Francfort qui n'a pas de modèle schématique la traduisant concrètement.

Les règles de la méthode que je présente ci-dessous, mènent à ce qui s'appelle : « la modélisation systémique ». La modélisation systémique, c'est la représentation graphique du système des échanges. Comme nous allons le voir, cette «modélisation » se fait à trois niveaux différents. Ces niveaux représentent les étapes de tout travail de recherche qualitative. Tout d'abord, on extrait, du fouillis de tous les échanges observables, des échanges remarquables par leurs récurrences et leurs similitudes. Dans un deuxième temps, on catégorise ces échanges analogues. Dans un troisième temps, on cherche la signification de chaque catégorie d'échange dans la totalité du système. Comme on le voit, la méthode de modélisation est délicate. Elle exige des chercheurs rompus aux analyses qualitatives. Ceci, de mon point de vue, explique largement qu'elle soit si peu utilisée.

\section{Première étape : la définition du « cadrage »}

La première étape de la technique systémique appliquée à l'étude des communications découle du deuxième principe vu ci-dessus : il convient, compte tenu de la problématique de recherche, de définir un niveau pertinent d'observation-enquête ; ou, réciproquement, compte tenu du niveau disponible d'observation-enquête, de définir les problématiques abordables. Il faut insister sur cette idée d'interdépendance de la problématique et du cadrage (Mucchielli, 2003). Précisons aussi tout de suite que la bonne problématique ou le bon cadrage de l'étude ne sont pas nécessairement trouvés immédiatement. Des allersretours avec le terrain permettent de mettre en œuvre la récursivité du travail de découpage de l'environnement menant à la bonne définition du cadre.

\section{Deuxième étape : L'observation des interactions fortes et constantes venant du contexte englobant.}

Le cadrage effectué délimite donc un sous-système dans un système plus vaste. Le système englobant le sous-système est rarement sans avoir des interactions avec ce soussystème. Il convient d'essayer de repérer ces interactions et d'en évaluer le poids pour le sous-système. Lorsque des interactions importantes viennent du système englobant sur le

Communication et organisation, $30 \mid 2006$ 
sous-système délimité par le cadrage, elles signalent presque toujours que la solution du problème rencontré dans le sous-système sera de l'ordre d'une intervention du système englobant sur le sous-système.

\section{Troisième étape : L'observation « hic et nunc " des "communications généralisées récurrentes" entre les acteurs.}

Pour arriver à une description de situation qui pourra permettre la modélisation systémique des communications, il nous faut observer tout ce qui peut prétendre être " communication ", c'est-à-dire qui peut prétendre vouloir dire quelque chose pour un acteur avisé. La mission est alors extrêmement difficile puisque, au départ, l'observateur n'est pas cet « acteur avisé ». En effet, qu'est-ce que cet « acteur avisé » ? C'est quelqu'un qui va comprendre un phénomène observable produit dans le champ de l'observation en tant que « communication qui veut dire quelque chose ». Ce phénomène observable aura un sens pour lui. Il sera «lu», il sera donc construit, par cet acteur avisé, en tant que communication. La communication dont nous parlons n'est donc pas définie par sa forme concrète (une parole, une attitude, une conduite,...), mais par sa nature: c'est un phénomène qui doit pouvoir " faire sens " pour quelqu'un. Dans les situations à observer, il faut se dire qu'il y a toujours un acteur avisé pour lequel tel ou tel phénomène expressif fait sens. Il faut aussi se dire que cet acteur n'est quasiment jamais seul à trouver telle ou telle signification à telle ou telle conduite expressive. En effet, dans les organisations surtout, les processus interprétatifs mis en oeuvre par les différents acteurs sont largement partagés et collectifs.

\section{L'observation des « non-communications »}

Dans l'étude systémique des communications, on prend en compte -en tant que communication- des "non-communications ", c'est-à-dire des choses qui auraient pu se dire ou se faire et qui ne se sont pas dites ou faites. Ce genre d'éléments d'observation ne saute pas aux yeux immédiatement, c'est souvent après coup, lorsque l'on a tracé les premiers traits fléchés du schéma systémique que l'on prend conscience que «cela pourrait se passer autrement ». On peut alors retourner à l'observation pour remarquer, qu'effectivement, cela ne se passe pas et que cela est même particulièrement évité. Il y a là une vraie difficulté de l'observation. Repérer de telles "non-communications" demande d'avoir des points de comparaison.

\section{La recherche des redondances d'échanges et des formes de ces échanges}

Tout observer ne met pas l'observateur en difficulté. En effet, s'il ne peut tout observer dans un laps de temps court, il peut prendre son temps car il est sûr que les choses qui lui ont échappé se présenteront de nouveau à son observation. C'est là la conséquence de l'existence du système des communications qui veut que les échanges soient " récurrents », c'est-à-dire reviennent régulièrement entre les acteurs. Il faut donc se concentrer sur les successions des échanges pour essayer de repérer des redondances dans des suites d'interactions perçues d'ailleurs, sous leurs " formes », pour formuler un scénario répétitif. Repérer les redondances dans les successions des échanges perçus sous leurs formes, est une rupture des habitudes d'observation. Il s'agit de faire porter son attention sur les cycles de la communication et non sur l'argumentation interne à un 
échange. Il faut montrer comment des «formes" d'expression se répondent toujours dans une même succession, plutôt que de montrer comment des contenus se répondent au niveau argumentatif ou du contenu- les uns aux autres.

\section{Quatrième étape : la schématisation des relations au niveau immédiat}

Dans cette deuxième étape, on s'efforce de porter sur un schéma les relations dites "concrètes et récurrentes" qui s'échangent entre les acteurs. Ces "communications concrètes et récurrentes" sont des relevés de propos, d'actions ou d'attitudes, effectivement perçues dans leur non-interprétation originelle. De fait, cette étape est imbriquée dans l'étape suivante. En effet, pour repérer une "récurrence ", il faut déjà faire un travail de généralisation et de catégorisation.

\section{Cinquième étape : l'explicitation des « formes » des échanges}

41 Ce sont les redondances repérées, dont nous parlions ci-dessus, qui permettent l'explicitation des formes des échanges. Ces formes étant donc des catégorisations formelles de communications. On refait donc le schéma des relations en portant ces formes relationnelles.

La forme d'un échange, c'est la catégorie générale du message qui est délivré à travers un ensemble de contenus concrets. Ainsi, dire: "Oh, là la, vraiment tu m'embêtes" et dire "Ils sont toujours en train de me faire des difficultés", c'est avoir la même "forme d'expression ", c'est se plaindre. On voit donc qu'une forme de communication (ou de relation) est une catégorie générale qui peut contenir de nombreuses expressions du même genre, c'est-àdire qui ont, dans le contexte et pour les acteurs, la même forme globale.

L'observation d'un système d'interactions s'apparente alors, comme le dit Watzlawick, à l'observation d'une partie d'échecs. Nous dirions plutôt qu'il a identifié un modèle complexe de redondances. Si la pente de son esprit l'y portait, il pourrait naturellement conférer un sens à chaque pièce et à chaque règle du jeu. Il pourrait forger toute une mythologie compliquée à propos du jeu et de sa signification "profonde » ou « réelle » notamment des histoires fantaisistes sur l'origine de ce jeu...mais tout ceci est superflu pour comprendre le jeu lui-même... On cherche à discerner la redondance dans l'interaction humaine. Concrètement, on commence par observer le système donné en action et on tente ensuite de définir les règles qui président à son fonctionnement. Nous dirions son "programme" par analogie avec un ordinateur. Un tel échange laisse ensuite percer sa signification si on le replace dans le contexte des interactions actuellement en cours (étape 4, ci-dessous, de la modélisation).

\section{Sixième étape : la contextualisation systémique}

Cette étape est l'étape fondamentale de la « contextualisation systémique ». C'est elle qui fait apparaître les significations à affecter aux formes des échanges. Dans cette étape, on reprend tour à tour, toutes les formes d'échanges ayant lieu entre les acteurs et portées sur le schéma précédent. On met chaque forme d'échange en regard de l'ensemble du système des formes de relations et l'on se demande quelle peut alors être la signification de cette forme relationnelle dans le contexte du système. 
En fait, ce qui est le plus important pour trouver les significations, en relation avec le contexte construit de la totalité des échanges, c'est d'expliciter le «jeu » global qui est joué par l'ensemble des acteurs. C'est l'accès aux enjeux partagés par les acteurs dans ce «jeu» qui donne la clé des interprétations. Lorsque l'on a fait la mise en relation de chaque forme d'échange avec les enjeux collectifs de fond des acteurs, on aboutit à un schéma qui porte l'ensemble des significations des échanges entre les acteurs. C'est ce schéma final qui peut être appelé la « modèlisation systémique finale ».

\section{Le rôle de la modélisation dans l'approche systémique qualitative des communications dans l'organisation}

\section{La modélisation comme hypothèse de fonctionnement}

L'ensemble de la procédure que nous venons de décrire représente le travail de la «modélisation systémique ». La méthode de l'analyse systémique ne s'arrête pas là. Après le travail de modélisation que j'ai rapidement évoqué (ou pendant ce travail, d'ailleurs), la construction des modèles donne lieu à des commentaires sur la compréhension de ce qui se passe entre les acteurs. L'ensemble de ces commentaires forme la partie analytique de la méthode systémique d'étude des communications. Les commentaires faits à partir de la réécriture finale des catégories signifiantes des échanges, permettent, en particulier, d'accéder à un raisonnement sur le fonctionnement du système.

La modélisation, en systémique qualitative, est une opération qui ne consiste pas à représenter le « modèle » de la théorie systémique. Ce modèle étant discursif, comme on l'a vu, il n'y a donc pas de schéma qui le représente. La «modélisation » est à prendre au sens où l'on s'efforce de représenter un fonctionnement concret et non une théorie. J.-L. Le Moigne a analysé cet effort que représente, dans les approches constructivistes, la modélisation. Pour lui, la modélisation est l'équivalent de l'hypothèse des sciences positivistes. En effet, on raisonne sur une modélisation comme on raisonne sur le résultat d'une expérience dans les sciences physiques et naturelles. L'expérience, en sciences positivistes permet de valider une hypothèse, le modèle, dans les sciences constructivistes, permet de vérifier la plausibilité et l'utilisabilité de la représentation du fonctionnement donnée par la modélisation.

\section{La modélisation réalisatrice d'une « contextualisation scientifique »}

Il nous faut insister sur le fait que le sens de la communication prise en compte dans ce «modèle», est sens par rapport au système dans lequel elle se déroule. Méthodologiquement, cela conduit à prendre le système de communications finalement explicité comme référentiel prioritaire de lecture du sens. Le sens de la communication faite n'est plus porté par son contenu, comme dans l'approche classique, mais il est "construit» en relation avec l'ensemble des communications se déroulant dans le système. Il s'agit là d'une " contextualisation » scientifique : on construit un contexte (le système), pour qu'il serve de support de fond à la lecture interprétative des formes d'échanges. C'est par rapport à ce contexte systémique construit que se fait la lecture du sens des divers échanges récurrents. La modélisation systémique apparaît bien comme une des méthodes constructivistes des sciences humaines. Le contexte systémique construit par l'analyste et le jeu collectif des acteurs (avec ses enjeux collectifs) sont, en 
règle générale, largement hors de portée de l'acteur tout venant qui reste centré sur chaque échange et sur les contenus des échanges.

\section{Exemple de début d'application de la modélisation systémique} de la méthode de modélisation). Ce "cadrage" est une des difficultés de l'application de la théorie et de son modèle, car, un cadrage, comme je l'ai dit, ne permet d'étudier que certaines problématiques et non d'autres, et chaque problématique (ici problématique $a$ priori de fonctionnement collectif), ne relève que d'un cadrage pertinent. Je vais donc, sur ce "constat" et son hypothétique problématisation, prendre le risque de découper une situation dans l'ensemble environnemental de l'entreprise, situation constituée d'un ensemble d'acteurs jugés potentiellement partie prenante. Je vois d'ailleurs, dès cette première démarche comment le "modèle systémique" est "ouvert" : il me laisse prendre des initiatives et je ne suis surtout pas obligé de "coller" au constat de départ qui me fixe a priori trois acteurs concernés. Dans l'exemple que nous avons pris, je vais donc considérer que de nombreux acteurs sont concernés et que mon enquête va progressivement me révéler les acteurs, internes à l'organisation qui sont également partie prenante.

En fait, je vais découvrir que les chefs de service et le directeur ne sont pas les seuls concernés et que toute l'équipe de direction est entraînée dans une sorte de dispute générale qui a des répercussions sur tous les personnels. Entraînés dans la dispute, ils se sont divisés en trois sous-ensembles : deux sous-groupes de partisans de chacun des chefs de service et un sous-groupe de spectateurs.

Pour trouver le "système" dans lequel les échanges repérés s'insèrent, je vais donc observer les acteurs définis par mon "cadrage" et je vais repérer les "échanges récurrents" entre ces acteurs (encore un concept de la théorie et du modèle). Il s'agit là d'une conséquence du troisième principe de la modélisation systémique qui me dit que pour accéder au "système des échanges" il faut passer par le repérage de "communications récurrentes". Autrement dit, mon observation est fortement guidée. Je ne vais pas observer et repérer toutes les communications ayant lieu entre les différents acteurs, mais seulement celles qui reviennent et se ressemblent dans leur "formes" (c'est là la définition du concept de "communications récurrentes"). Pour ce faire, je vais faire des interviews mais je ne pourrai en rester aux récits des sujets. Il me faudra trouver des "incidents critiques" (concepts de l'approche) sur lesquels je ferai parler tous les acteurs 
pour essayer d'avoir un point de vue cohérent et synthétique, débarrassé des interprétations subjectives des uns et des autres. Ce travail d'enquête quasi policière, m'oblige d'ailleurs à repérer des "formes" d'échanges plutôt que des "contenus" d'échanges et ceci est très positif puisque, comme nous le savons, j'aurai à faire une modélisation du système en ne retenant que les catégories formelles des échanges récurrents repérés.

\section{Le constructivisme de l'approche par la systémique qualitative}

Que l'analyse systémique soit "constructiviste", cela semble aller de soi. En effet on considère que c'est l'école de Palo Alto qui a vraiment apporté cette analyse dans les sciences humaines. Or, cette école est dite "constructiviste». Watzlawick et ses coéquipiers ne montrent-ils pas que les malades qu'ils traitent à travers des «injonctions paradoxales » et des « communications de recadrage » se font une idée personnelle de la situation, qu'ils « construisent » des réalités secondaires? L'école de Palo Alto n'a-t-elle pas produit l'ouvrage: "La réalité de la réalité. Introduction au constructivisme »? Certes, ceci est vrai. Mais l'école de Palo Alto est à la source d'une confusion entre le "constructionnisme" des acteurs sociaux et le "constructivisme" de l'analyse systémique qualitative. Les auteurs de l'école de Palo Alto n'ont jamais vraiment insisté pour montrer comment leurs analyses étaient constructivistes. Ils ont bien montré, par contre, comment les individus et les groupes construisaient les réalités dans lesquelles ils vivaient. Or, au niveau des acteurs sociaux, cette « construction» des significations des éléments des situations de vie est du «constructionnisme». Le constructivisme de l'analyse systémique qualitative ne repose pas sur l'idée de "construction sociale de la réalité sociale par les acteurs sociaux ", mais sur la démonstration que sa méthodologie générale obéit aux principes du constructivisme scientifique. Nous allons démontrer cela en passant en revue les rapports de la méthode avec les principes forts de l'épistémologie constructiviste.

Le principe téléologique : « on ne peut pas séparer la connaissance construite des finalités attachées à l'action de connaître ».

Pour les théoriciens du constructivisme, il faut absolument considérer que l'acte cognitif tout entier (celui qui conduit à la connaissance) a un caractère intentionnel et, donc, qu'il est finalisé. La connaissance construite par cet acte dépend de la finalité qui a été à la base de son explicitation. La connaissance, pourrions-nous reformuler, dépend donc de la finalité de l'acte intellectuel qui y mène (Von Glasersfeld, 1988, p. 35).

57 L'analyse systémique qualitative des échanges respecte ce premier principe. Elle est orientée par deux finalités. La première est celle de la mise en avant du sens dans l'explicitation des systèmes d'échanges. Ce qu'elle va mettre en lumière ne peut être détaché de cette volonté de mettre en exergue les significations collectives de ces communications. La deuxième finalité qui oriente le diagnostic sur les communications organisationnelles fait avec l'approche de la systémique qualitative, c'est la recherche d'une compréhension en vue d'une intervention pour améliorer les choses. Le diagnostic systémique reprend là l'orientation thérapeutique de ses origines palo altienne.

Le principe de l'expérimentation de la connaissance : «la connaissance est totalement liée à l'activité expérimentée et donc vécue du sujet ». 
59 Pour le constructivisme, le réel connaissable est un réel phénoménologique, celui que le sujet connaissant expérimente et qu'il construit à travers des représentations symboliques (schémas, lettres, chiffres, phonèmes, ...). Ainsi, pour le constructivisme, nous ne pouvons en aucun cas concevoir un monde indépendant de notre expérience.

60 Dans l'analyse systémique qualitative, ces représentations symboliques seront les "systèmes" construits à base de ronds et de flèches étiquetées. Dans l'approche systémique, nous avons bien cet engagement du chercheur dans des confrontations directes ou empathiques, avec les objets de la connaissance qu'il propose : les acteurs, les relations, les boucles d'interaction, les formes relationnelles, les significations des échanges... Ces objets ne peuvent être pré-connus dans leurs modalités particulières et définitives avant cette confrontation expérientielle. Cependant, ils sont, au départ de l'investigation, connus dans leurs formes conceptuelles : les «macro-concepts » (Morin, 1990). Cela veut dire que le chercheur sait, par exemple, qu'il doit trouver un "système d'échanges ", mais il ne sait pas lequel concrètement parlant; il sait qu'il doit trouver des " boucles de récursivité » entre les acteurs, mais il ne sait pas lesquelles; il sait qu'il doit trouver des interactions signifiantes, mais il ne sait pas quelles seront leurs formes concrètes, ... La « construction par l'expérimentation ", c'est alors le travail de précision qui fait que de tels "macro-concepts", définis dans leurs formes générales, soient précisés jusqu'à prendre leurs formes définitives dans la modélisation systémique finale.

Le principe de la connaissance par l'interaction: "la connaissance est le fruit d'une interaction du sujet connaissant et de l'objet de connaissance ».

Il découle de ce qui a été dit précédemment, que l'on connaît scientifiquement, par et dans l'interaction. La connaissance exprime l'intelligence de l'expérience du sujet connaissant, et cette interaction du sujet et de l'objet qu'elle représente. Le sujet ne connaît pas de "choses en soi », mais il connaît l'acte par lequel il perçoit l'interaction entre les choses. Il ne connaît pas cet arbre, mais l'interaction de cet arbre et de son contexte. «L'intelligence (et donc l'action de connaître) ne débute ni par la connaissance du moi, ni par celle des choses comme telles, mais par celle de leur interaction; c'est en s'orientant simultanément vers les deux pôles de cette interaction qu'elle organise le monde en s'organisant elle-même" (Piaget, 1980, p.311). Rappelons nous que les constructivistes disent que « l'on ne connait pas l'arbre, mais l'interaction de l'arbre avec son contexte ». En disant : « il ne connaît pas cet arbre, mais l'interaction de cet arbre et de son contexte ", les constructivistes prennent ici un parti pris phénoménologique et contextualisant. En effet, de l'interaction pensée entre les éléments du monde naissent les significations. Le monde à connaître est un monde de relations entre les choses à connaître. Il faut rechercher des « interactions » et faire surgir, à travers cette recherche, des significations. C'est donc un "univers de significations » qu'il faut s'efforcer de construire (Berger et Luckmann, 1986). On sait, par ailleurs que cet univers est une "représentation» du monde parmi les autres représentations possibles. L'approche systémique qualitative et la méthode de la modélisation systémique satisfont bien à ce programme de contextualisation pour faire émerger les significations. En effet, comme nous l'avons vu, pour construire la modélisation finale, celle qui porte les significations, il faut lire chaque échange dans le contexte du système explicité et de sa logique sousjacente. Il s'agit donc bien d'un processus de contextualisation.

63 Le principe de la récursivité de la connaissance : " la connaissance établie, et le processus de connaissance qui l'établit, se structurent réciproquement ». 
64 j'organise le monde. La connaissance, pour le constructivisme, est le processus de sa propre construction. Pour le constructivisme, la connaissance n'est pas indépendante de ce qu'elle élabore (et inversement). Il y a récursivité de ce qui est en train de se construire sur les processus de la construction elle-même. La connaissance est un processus actif produisant le résultat. Elle est autant opérateur qu'opérande. Le caractère récursif de la connaissance des phénomènes rend compte de l'interdépendance assumée entre le phénomène perçu et sa connaissance.

Le principe de la récursivité de la connaissance exclut toutes les méthodologies «fermées ", c'est-à-dire incapables de souplesse et d'un minimum d'adaptabilité aux résultats qui sont progressivement construits. Il privilégie toutes les méthodologies itératives et modulables. Ces méthodologies de "projet », où, au fur et à mesure de l'avancement, on modifie la procédure pour l'adapter au résultat émergent. Du point de vue des techniques de recueil et d'analyse, la recherche «constructiviste » doit puiser dans les techniques de recueil non "mécaniques ", c'est-à-dire non fixées une fois pour toutes. Une grille d'analyse ne peut être utilisée telle quelle tout au long d'une même recherche. Elle doit pouvoir être adaptée progressivement. Le chercheur a donc la nécessité de prendre des instruments "généraux", modulables et extensibles. L'application brute d'une théorie forte et de sa méthodologie canonique définie semble exclue. En effet, dans l'application d'une théorie, il n'y a pas cet aller-retour créatif entre la méthode et le résultat. Le résultat est connu dans sa forme globale (il vérifie la théorie); la méthodologie est verrouillée (elle est l'application de procédures menant à une forme globale de résultat connu). Dans la recherche constructiviste, la construction scientifique doit fonctionner comme l'intelligence humaine, c'est-à-dire s'adapter sans cesse à la construction faite. Les méthodes d'élaboration des résultats scientifiques doivent être flexibles et être reliées aux construits obtenus. Ceci fixe le genre de méthodologie de recueil et d'analyse à utiliser. Ce sont des méthodes fondées sur l'intuition et l'adaptation intelligente à ce que l'on découvre. Dans ces méthodes, l'enquêteur est nécessairement actif intellectuellement dans le maniement de l'instrument de recueil qu'il utilise puisque cet instrument le met en contact, physique ou intellectuel, direct avec son objet d'étude. Par ailleurs, il doit sans arrêt réfléchir et intervenir pour orienter sa quête en fonction des résultats progressifs de ses analyses.

La théorie systémique qualitative et la méthodologie de la modélisation des relations qui va avec, sont des entités scientifiques souples et larges. La théorie ne fait que dire de rechercher des «systèmes d'interactions " (des boucles de récursivité, des logiques de jeu,...) et ne fait que donner, ensuite, un principe large d'intervention : « on peut agir sur ces systèmes par recadrage ». Elle ne précise rien de plus et laisse donc beaucoup d'inventivité au chercheur. Dans la modélisation systémique, il existe une relation entre la conduite de la recherche et le contexte systémique final convoqué pour l'interprétation. "L'activité de recherche » et le "contexte interprétatif » sont des éléments qui s'élaborent et se déterminent mutuellement dans une équation simultanée que le chercheur essaie de résoudre afin de définir la nature des événements qu'il veut analyser. L'activité de recherche porte des orientations et des attentes qui font surgir un contexte pertinent, lequel contexte modifie les orientations de la recherche et ainsi de suite. L'émergence du contexte interprétatif pertinent (le système), se fait donc en grande partie, sous l'impact de l'activité de recherche et du contexte lui-même (que celui-ci soit défini a priori ou qu'il soit émergent).

Communication et organisation, 30 | 2006 


\section{Les limites du modèle et de la modélisation systémiques}

67 J'ai déjà évoqué la principale limite d'une étude utilisant ce modèle et la modélisation qui va avec: il s'agit de la difficulté d'utilisation d'une telle approche. C'est d'abord une approche qualitative qui demande des chercheurs expérimentés. C'est ensuite une approche constructiviste, qui demande des chercheurs capables de jouer le jeu de ce type d'approche.

68 En ce qui concerne les difficultés liées à la maîtrise des techniques de l'approche qualitative nécessaire, je soulignerai :

- la difficulté du repérage des échanges « qui se ressemblent » et concernant le problème, dans la masse des échanges à observer ;

- la difficulté de définir progressivement le bon cadrage, lorsque l'on part d'un problème organisationnel donné à traiter ;

- la difficulté à ne pas tout de suite interpréter les échanges et leurs " formes ", en fonction de théories toutes faites psychosociologiques ou sociologiques ;

- la difficulté de la catégorisation des échanges analogues. Cette catégorisation devant mettre sur la piste des enjeux collectifs sous-jacents ;

- la difficulté de la saisie de la « logique » du jeu collectif (ou de la formulation d'une synthèse sur l'enjeu collectif principal des acteurs);

- la difficulté de la formulation des significations des échanges.

- En ce qui concerne les difficultés liées à la maîtrise des procédures constructivistes, je soulignerai :

- la difficulté de la fameuse « expérimentation intellectuelle » des macro-concepts et de leur confrontation avec la réalité pour les « concrétiser »;

- la difficulté de la mise en oeuvre concrète de la « connaissance par l'interaction », demandée par la méthodologie constructiviste. Il s'agit, en fait, d'appréhender intuitivement les significations sur le terrain de l'enquête pour, ensuite, et ensuite seulement, les vérifier dans la modélisation.

69 La méthode permet d'arriver à des résultats analytiques extrêmement riches. Ils sont d'ailleurs à la base des résultats très probants acquis par son utilisation en consultance pour la résolution des problèmes humains de fonctionnement. Mais elle est très exigeante. Les chercheurs n'aiment pas suivre des "procédures » et se plier à une telle ascèse intellectuelle de manipulation et de réduction de macro-concepts. Ils préfèrent aller vite dans le recueil et interpréter rapidement.

70 Il faut aussi souligner que cette méthode est difficile à enseigner. Les « cas » que l'on peut fabriquer pour ce type de formation ne permettent pas aux apprenants de se confronter aux réelles difficultés des enquêtes de terrain.

\section{Un modèle « situationnel » pour le diagnostic des communications organisationnelles}

Nous pouvons ici répéter ce que nous avons dit pour la théorie systémique qualitative d'étude des communications. J'irai donc plus vite pour cette présentation. La théorie 
"situationnelle" de référence est une théorie dite "lâche". Elle s'appuie sur des principes d’une grande généralité que nous allons rappeler.

\section{Les principes de la théorie de référence}

72 Principe 1: Les communications (dans les organisations comme ailleurs), prennent leurs significations dans le contexte de la situation dans laquelle elles ont lieu.

Principe 2: Une situation de communication, pour la commodité des recherches, est décomposable en contextes dont les quatre principaux sont: le contexte des normes, le contexte des enjeux, le contexte des positionnements et le contexte de la qualité des relations entre les acteurs.

Principe 3: Une situation, d'un point de vue objectif collectivement partagé, cela existe rarement. Une situation est toujours plutôt une "situation-pour-un acteur ». Cela veut dire que, compte tenu de ses valeurs et normes, de ses enjeux, de sa position et de son statut, de sa place dans le réseau des relations, chaque acteur produit une définition personnelle de la « situation-pour-lui ». De ce fait, une communication, faite par tel ou tel acteur, ne prend plus nécessairement la même signification pour les différents acteurs en présence dans la situation.

Principe 4: Une "situation-pour-un-acteur", contient toujours une problématique principale à laquelle il s'efforce de répondre à travers ses activités expressives (communications, attitudes, conduites en actes, ...). Si la situation est interprétée différemment par les différents acteurs, le problème est aussi perçu différemment. De ce fait, dans les organisations où de nombreux problèmes standards et récurrents doivent sans arrêt être réglés, les risques d'incompréhension et de conflits entre les acteurs sont importants.

76 Principe 5: Les définitions de la situation, données par les différents acteurs, sont accessibles à travers leurs conduites (qui sont logiques par rapport à leur vision des choses) et à travers leurs autres expressions : commentaires et échanges divers.

77 Principe 6: Les dysfonctionnements de la communication dans les situations organisationnelles sont en grande partie imputables aux définitions différentes de la situation données par les différents acteurs. Les "communications" dont il s'agit sont aussi bien des échanges de travail entre les acteurs que des directives globales données par les dirigeants. La compréhension des points de vue des acteurs sur la situation permet de poser les bases d'un diagnostic qui permettra ensuite des interventions orientées.

\section{Le modèle théorique}

78 Le modèle représentant la théorie est un schéma traduisant les principes ci-dessus. Le schéma montre qu'une situation peut être perçue de différents points de vue. Chaque point de vue dépend, pour chaque acteur, de ses normes, de ses enjeux, de sa position et de la qualité des relations entretenues avec les autres, de ce qui s'est passé entre les acteurs, .... Chacun accède donc à une vision, que l'on peut considérer comme partielle, de la situation totale.

Fig. 2 Les visions différentes de la situation par les acteurs de l'organisation 
D.R.H

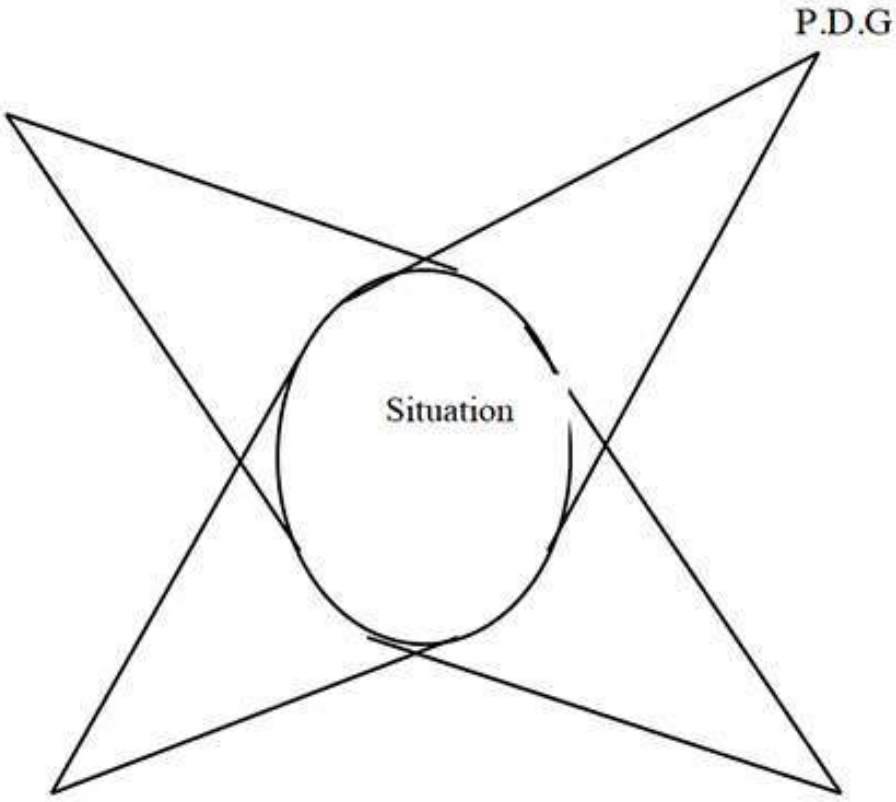

Ingénieur d'Etude

Contremaître (supposons qu'il soit très centré sur l'efficacité et la rationalité et que le D.R.H. soit plus centré sur la prise en considération des problèmes humains et sur le "relationnel"). Ce même P.D.G., en ce qui concerne la situation examinée et les problèmes qu'elle contient, n'a pas, non plus, les mêmes enjeux que le D.R.H. (supposons qu'il ait, comme enjeu prioritaire, d'assurer le développement de l'entreprise dans un créneau opportun, quoi qu'il en coûte et que le D.R.H. soit avant tout préoccupé de la capacité de résistance au stress des personnels)... Ces deux acteurs ne partagent, comme le modèle le montre, qu'une toute petite partie de la définition de la situation et de son problème. Le même raisonnement peut être étendu aux autres acteurs de la situation. L'Ingénieur d'Etude et le Contremaître ont aussi leurs visions personnelles et différentes des choses, différentes entre eux et différentes des visions des deux autres acteurs.

\section{Les présupposés du modèle}

Le présupposé majeur du modèle (l'existence de définitions différentes de la situation collective par les différents acteurs), entraîne d'autres présupposés. Le modèle contient, en particulier, une définition implicite de la communication organisationnelle, celle qui va se faire entre les acteurs pour aboutir à des activités coordonnées de collaboration et de résolution de problèmes. La communication apparaît comme l'ensemble des activités d'échange qui va permettre aux acteurs de rapprocher leurs points de vue, pour trouver une définition commune de la situation et de son problème, et pour mettre en place, ensuite, des activités collectives de résolution de problème organisationnel. La communication qui se mettra en place n'est pas forcément de forme participative, laisse la place à des jeux d'influence. Elle peut aussi très bien être une communication 
descendante autoritaire, venant de la direction, laquelle peut imposer sa définition de la situation, du problème et de la manière de le traiter.

Le modèle se complète donc, comme je l'ai signalé dans les généralités sur les modèles, par un raisonnement sur son fonctionnement. Dans notre cas, les acteurs sociaux, à travers un dialogue qui peut être sincère, peuvent arriver à construire collectivement une définition partagée de la situation-problème. Mieux même, au cours de leurs échanges, des idées nouvelles sur cette situation peuvent émerger. Le processus de la communication les conduit à arriver à un point de vue commun représenté par le point Oméga sur le schéma ci-dessous.

Fig. 3. Processus de construction collective de la vision commune

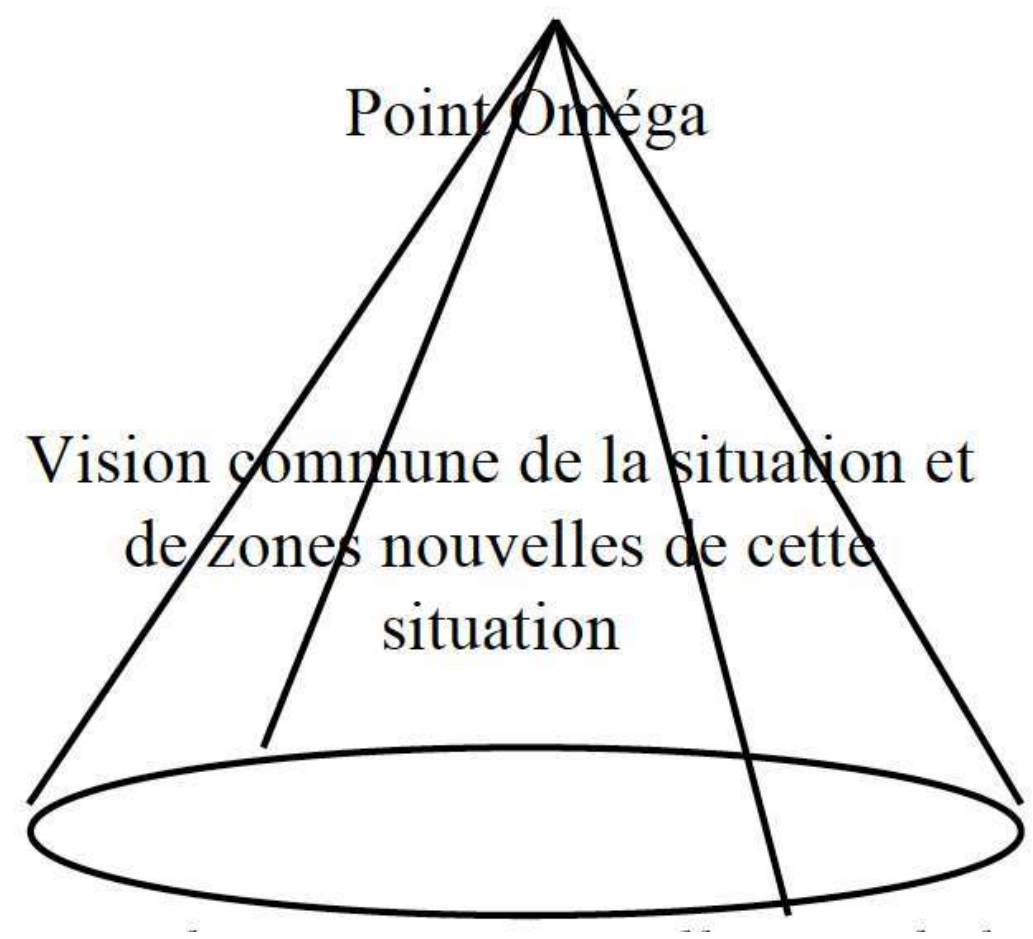

\section{Une modélisation de la situation collective}

La théorie situationnelle et son modèle de discussion se prolongent par une véritable « modélisation » de la situation collective. Cette modélisation se réalise sous la forme d'un « tableau panoramique » qui est renseigné à partir d'une enquête de terrain.

Ce qu'il est intéressant de voir, c'est que le tableau vide, fonctionne comme un " guide d'enquête ». En effet, pour le renseigner complètement, il va falloir aller chercher sur le terrain des données pertinentes. Ces données ne sont pas n'importe lesquelles. Elles concernent, pour une situation donnée et pour chaque acteur, ses valeurs et normes, ses enjeux, ses positionnements et les qualités des relations qu'il entretient avec les autres (conséquence du principe $2 \mathrm{du}$ modèle théorique). Remarquons que les têtes de colonne du tableau portent des «macro-concepts ». La méthode de renseignement du tableau nous place d'une manière privilégiée dans une approche qualitative et constructiviste. En effet, par exemple, on sait que l'on doit trouver les « normes » des différents acteurs (ou 
leurs enjeux, ...), mais, bien entendu, la formulation concrète de la norme (ou de l'enjeu) en question, sera le résultat du travail du chercheur et de la procédure constructiviste mise en oeuvre.

Grille d'enquête ou tableau de modélisation de la situation

\begin{tabular}{|l|l|l|l|l|}
\hline $\begin{array}{l}\text { Eléments } \\
\text { Acteurs }\end{array}$ & Normes & Enjeux & Positionnement & Qualité des relations \\
\hline Acteur 1 & & & & \\
\hline Acteur 2 & & & & \\
\hline Acteur $\mathrm{n}$ & & & & \\
\hline
\end{tabular}

$84 \mathrm{Au}$ fur et à mesure que se déroule le renseignement du tableau, on élabore donc une véritable modélisation de la situation collective dans laquelle se trouvent les différents acteurs. Le tableau est d'ailleurs dit "panoramique", car il donne au chercheur, une vision globale comprenant les différentes définitions de la même situation, faites par les différents acteurs. Cette "modélisation » est, bien entendu, comme toute modélisation, une simplification de la « réalité » situationnelle complexe dont il faut rendre compte. En effet, elle sélectionne un ensemble limité de données, dites pertinentes pour le modèle : celles qui concernent le tableau justement.

Ce tableau est issu du tableau inventé par "l'analyse actionniste» de la sociologie anglaise des années 70 (Silverman, 1973). Dans le tableau de Silverman, la première colonne porte sur la " vision de la situation de l'acteur considéré »; la deuxième colonne, sur les « actions typiques » faites par les différents acteurs de cette colonne; la troisième colonne, sur les "significations attachées par l'acteur à ses actions typiques"; la quatrième colonne, porte sur les «implications de l'acteur dans la situation»; la cinquième colonne, porte sur les «conséquences voulues des actions » et la dernière colonne, sur les « conséquences non voulues de ces mêmes actions » (Mucchielli et Noy, 2005). Les concepts utilisés dans cette modélisation sont propres à la "théorie actionniste " proposée par Silverman. La réalisation de tels "tableaux synoptiques » de représentation des situations organisationnelles, nous ouvre des portes nouvelles pour la modélisation du "contexte organisationnel», qui, comme on le sait, est le contexte fondamental dans lequel les activités des acteurs sociaux prennent un sens dans l'organisation (Le Moënne, 2003). On peut effectivement penser à utiliser des tableaux qui font ressortir, dans les colonnes, des éléments pertinents du contexte organisationnel différents de ceux proposés par ces deux approches. Ces éléments pertinents étant choisis en fonction du problème managérial ou de fonctionnement à traiter.

Une fois le tableau de la modélisation de la situation pour les acteurs entièrement rempli, on peut le commenter et raisonner dessus. On le fait alors fonctionner, comme pour la modélisation systémique, comme une "hypothèse » sur ce qui peut se passer si l'on décide ceci ou cela (selon la formule de Le Moigne). En effet, toute décision d'intervention ou d'action d'un des acteurs en cause, prendra un sens, pour les autres acteurs, dans la situation explicitée par cette modélisation puisque l'on a bien formulé ce qui constituait la situation pour chacun des acteurs. On pourra, par exemple, réfléchir à un «plan de 
communication interne ", en calculant les réactions que chacun de ses éléments pourra provoquer chez les acteurs. Ces réactions sont calculées en fonction de ce que ces éléments prennent comme significations, dans le contexte de la situation pour chaque acteur. Ce raisonnement est alors une partie de ce qu'il est convenu d'appeler : « l'analyse situationnelle ».

\section{Le constructivisme du modèle}

Nous dirons que la méthode d'analyse situationnelle par le tableau panoramique est une méthode constructiviste, car elle satisfait aux principes forts du constructivisme. Nous allons démontrer cela en passant en revue rapidement les relations de la méthode avec les principes essentiels de l'épistémologie constructiviste.

Le principe téléologique: «on ne peut pas séparer la connaissance construite des finalités attachées à l'action de connaître ».

Nous avons vu que, dans une optique constructiviste, la connaissance dépendait de la finalité de l'acte intellectuel qui y menait. L'analyse d'une situation faite avec le modèle « situationnel » respecte ce principe. Elle est en effet orientée par la volonté de faire un diagnostic de la situation. Ce diagnostic devant ensuite fonder, non seulement la compréhension de la totalité de la situation, mais aussi la possibilité de prescrire des interventions, compte tenu des problèmes d'interprétation que l'on aura fait apparaitre. La recherche faite à partir de l'utilisation de ce modèle n'est pas "purement scientifique ». Elle est «entachée » d'utilitarisme. Le modèle sera donc, sans doute, plus utile à des consultants en organisation, ou à des managers eux-mêmes, qu'à des universitaires. Il n'a cependant pas à être ostracisé par les puristes de la « vraie » science, il a seulement à être reconnu pour ce qu'il est.

Le principe de l'expérimentation de la connaissance : «la connaissance est totalement liée à l'activité expérimentée et donc vécue du sujet ».

91 Pour le constructivisme, le réel connaissable, avons-nous dit, est un réel « expérimenté ». Par principe, dans cette épistémologie, nous ne pouvons en aucun cas concevoir un monde indépendant de notre « expérience ». Dans l'utilisation du modèle situationnel, il y a une expérimentation intellectuelle des macro-concepts. Ils doivent être connus, au préalable, dans leurs définitions théoriques, mais il faut les mettre à l'épreuve du terrain pour leur trouver des «formes concrètes». Le chercheur se confronte " expérientiellement » au terrain, en y recherchant des "normes ", des « enjeux », des "positions d'acteurs» et des "qualités de relations» entre les acteurs. Son objet de connaissance n'est pas la "norme », en général, par exemple, mais les formes concrètes prises par ce macro-concept, pour les différents acteurs. Comme pour la recherche d'un " système d'échanges ", le chercheur connait l'idée générale de ce qu'il doit trouver, mais il n'en connaît pas l'émergence concrète qui résultera de sa confrontation avec le terrain. Comme pour les macro-concepts utilisés dans l'analyse systémique qualitative des communications, nous pouvons dire que les macro-concepts, dont se sert au départ le chercheur sont des «savoirs institutionnalisés ». De ce fait, le chercheur est autorisé à utiliser ces outils conceptuels pré-donnés pour sa recherche. Ils sont censés être le résultat de son expérimentation intellectuelle de chercheur. La «construction par l'expérimentation ", voulue par la conception constructiviste, c'est alors le travail de 
précision qui fait que de tels "macro-concepts", définis dans leurs formes générales, sont précisés jusqu'à prendre leurs formes définitives dans l'analyse situationnelle finale.

Le principe de la connaissance par l'interaction: «la connaissance est le fruit d'une interaction du sujet connaissant et de l'objet de connaissance ».

Pour illustrer ce principe, nous avons dit que le sujet ne connaît pas de "choses en soi ", mais qu'il connaît à travers une interaction entre les choses à connaitre. Par exemple, disions nous, il ne connaît pas cet arbre, mais l'interaction de cet arbre et de son contexte. L'interaction menant à la connaissance est donc une "contextualisation » menée intellectuellement. Dans l'application du modèle situationnel, c'est bien à ce type d'activité que va se livrer le chercheur. Prenons encore l'exemple de la recherche de la norme de conduite d'un acteur. La forme concrète de la norme ne va pas lui apparaître comme " une donnée en soi », émergeant du terrain. Pour trouver cette norme, il va être obligé de contextualiser un ensemble d'indices comportementaux afférents au sujet observé. C'est à travers ce travail de mise en interaction qu'il va accéder à la compréhension de la norme mise en jeu. Ce type de travail intellectuel, il va le renouveler pour tous les éléments à mettre dans le tableau. Nous avons déjà souligné combien cette construction contextualisante se distingue des contextualisations primaires spontanées des acteurs en situation. C'est donc bien un univers de significations scientifiques que le chercheur construit par son travail intellectuel. Ce travail, comme nous l'avons vu, intègre toujours divers va-et-vient de « documentation » (au sens ethnométhodologique) et de complémentation et de comparaison avec d'autres phénomènes concomitants.

Le principe de la récursivité de la connaissance : « la connaissance établie, et le processus de connaissance qui l'établit, se structurent réciproquement ».

Nous avons vu que du point de vue des techniques de recherche, le "constructiviste» devait puiser dans les techniques de recueil non " mécaniques ", permettant des allers et retours conceptualisation-terrain. Nous avons vu que le chercheur avait la nécessité d'utiliser des instruments " généraux ", modulables et extensibles. C'est à cette condition qu'il peut exister des allers-retours créatifs entre la méthode et le résultat. Ici, dans l'utilisation du modèle de la théorie "situationnelle ", le résultat est connu dans sa forme globale : c'est le tableau renseigné. Ce tableau permet, bien entendu, de vérifier la théorie, puisqu'il la postule dans sa forme même dès le départ: il est rempli des normes, des enjeux, des positions et des qualités des relations entre les acteurs. Il existe cependant une certaine "récursivité » dans la construction de cette connaissance de la situation. Elle vient du fait que les macro-concepts donnés comme cadre de l'observation et de l'enquête sont flous et larges. On doit chercher des «normes ", mais il y a tellement de normes existantes, que la consigne laisse bien de la place à une construction récursive. Un certain nombre d'indices vont faire penser à une norme précise. On va la mettre dans le tableau. Mais il faudra la valider tout au long de l'enquête au fur et à mesure que d'autres indices seront recueillis. Ces indices confirmeront-ils ou infirmeront-ils la norme? Le retour au terrain va aussi permettre de chercher des indices nouveaux de validation de cette norme. En ce sens, la connaissance établie va structurer, en retour, le terrain. Comment ces indices permettent-ils d'affiner cette norme (structuration, retour venant du terrain) et comment ces précisions sont-elles ensuite confirmées dans la suite de l'enquête? Le chercheur fonctionne donc avec ces allers-retours permanents. Le modèle « situationnaliste » vérifie bien le principe de la récursivité des connaissances exigé par la posture constructiviste. 


\section{Intérêts et limites de la modélisation situationnelle}

96

l'étude des situations organisationnelles. Son utilisation en séminaire de formation est
performante pour diverses raisons. Elle force les participants à quitter leur vision interprétative immédiate du cas avec les solutions arbitraires immédiates qu'ils ont tendance à donner. Elle exige d'eux qu'ils distinguent bien ce qui est objectivement rapporté dans le cas de ce qui est "construit" par eux pour comprendre les différents points de vue des acteurs.

acteur à la situation (la "même"), pour un autre acteur. Elle forme donc à l'effort empathique, en montrant que c'est une potentialité de notre "nature humaine" (un vrai scandale de la connaissance philosophique, disait Schultz). La méthode montre que ces efforts d'empathie sont à notre portée dès que nous abandonnons nos préjugés (la fameuse "époché» de la phénoménologie) et que nous nous référons essentiellement à une intuition fondée sur notre acculturation. Elle fait toucher du doigt que les descriptions, constituant le fond du cas rapporté, peuvent être incomplètes et qu'il est relativement facile de les compléter, si l'on sait ce que l'on cherche. stratégies d'intervention. Celles-ci prennent en compte des objectifs, et surtout prennent en compte le fait que la communication peut être pensée comme une ressource d'intervention, du moment qu'elle s'appuie sur une connaissance des différentes visions de la situation. Une telle méthode apparaît comme une des bases d'une formation humaniste aux sciences humaines et à la consultance.

Comme pour la modélisation systémique des communications, je dirai que cette méthode de modélisation "situationnelle» est une méthode délicate. C'est une approche qualitative qui, comme toutes les méthodes qualitatives, demande des chercheurs expérimentés. C'est ensuite une approche constructiviste qui demande des chercheurs désireux de mener à bien une telle approche qui se trouve tout de même en rupture avec pas mal d'habitudes d'enquête.

\section{Conclusion : la contextualisation scientifique}

100 En conclusion, j'attirerai surtout l'attention sur le fait que les deux méthodes présentées sont des méthodes de construction scientifique de contextes interprétatifs. Ce sont donc des méthodes de "contex-tualisation scientifique» qui sont faites pour que les chercheurs formulent des interprétations les moins critiquables possible, puisqu'elle sont issues de procédures vérifiables, pouvant mener à l'accord des esprits.

101 Les constructions sont scientifiques, car elles se réfèrent à des procédures codifiées que tout chercheur peut mettre en oeuvre. Les constructions sont aussi scientifiques, car elles font appel à des concepts scientifiques connus de la communauté scientifique des sciences humaines : communication récurrente, catégorisation, enjeu collectif, vision du monde, enjeu, normes,... La démarche est encore scientifique dans le fait qu'elle part de macro-concepts et qu'elle fait arriver à leurs spécifications. Ce travail intellectuel est tout à fait classique en sciences humaines.

Communication et organisation, $30 \mid 2006$ 

interprétations est d'une nature différente des contextualisations que la plupart des chercheurs ont l'habitude de faire. Les contextualisations habituelles se font par rapport à des théories, plus ou moins implicites, et à leurs modèles schématiques. Une analyse, dans les termes de la sociologie française des organisations, par exemple, se fait en référence à la théorie de la lutte pour le pouvoir et à ses quelques concepts clés. Cette théorie est alors l'arrière plan, non construit empiriquement, mais donné a priori et immédiatement, par rapport auquel les phénomènes sont décodés. Les approches présentées distinguent nettement, ce qui est des a priori théoriques, et ce qui est de la contextualisation menant aux interprétations permises par l'acceptation des principes théoriques. La distinction de ces deux moments de la recherche scientifique en sciences humaines, me parait être une bonne chose pour la clarification du travail interprétatif dans les sciences humaines et pour le positionnement de nos disciplines.

\section{BIBLIOGRAPHIE}

Bachelard S., Quelques aspects historiques des notions de modèle et de justification des modèles, Actes du colloque Elaboration et justification des modèles, P. Delattre et M. Thellier, Maloine-Doin, 1979 , tome 1.

Bateson G., Vers une écologie de l'esprit, Paris, Seuil, 1977.

Berger P. et Luckman T., 1966, La construction sociale de la réalité, Paris, Méridiens Klincksieck, 1986.

Becker H.S., Outsiders. Etudes de sociologie de la déviance, Paris, Métaillé, 1985.

Bernard F., Un constructivisme « limité » pour la communication des organisations, in La place du constructivisme pour l'étude des communications, actes du colloque de Béziers, CERIC, Montpellier, éd., 2003, pp. 52-68.

Blin Th., Phénoménologie et sociologie compréhensive. Sur Alfred Schutz, Paris, L’Harmattan, 1995.

Boudon R., La logique du social, Paris, Hachette, 1979.

Bulle N., Modèle en sciences humaines, in Le dictionnaire des sciences humaines, Paris, PUF, sous la dir. de S. Mesure et P. Savidan), 2006, pp. 781-784. 
Coulon A., Situation (définition de la), article du Dictionnaire des méthodes qualitatives en sciences humaines et sociales, Paris, Armand Colin, 2005, pp. 255-256.

De Quieroz J.-M. et Ziotrovski M., L'interactionnisme symbolique, Presses de l'Univ. de Rennes, 1994.

De Fornel M. \& Quéré L., Situer la situation, in La logique des situations. Nouveaux regards sur l'écologie des activités sociales, Paris, éd. de L'Ecole des Hautes Etudes en Sciences Sociales, 1999, pp. 7- 32.

De Rosnay J., Le macroscope, Paris, Seuil, 1975.

Descombes V., Les institutions du sens, Paris, éd. de Minuit, 1996.

Dubet F., Sociologie de l'expérience, Paris, Seuil, 1994.

Elias, N., 1970, Qu'est-ce que la sociologie?, Paris, éd. de l'Aube, 1991.

Fornel M. et Quéré L., La logique des situations. Nouveaux regards sur l'écologie des activités sociales, Paris, éd. de l'EHESS, 1999.

Giorgi A., De la méthode phénoménologique utilisée comme mode de recherche qualitative en sciences humaines : théorie, pratique et évaluation, In J. Poupart et al. : La recherche qualitative, enjeux épistémologiques et méthodologiques, pp. 341-363, Montréal, Gaétan Morin Éditeur, 1997.

Glasersfeld Von E., 1981, Introduction à un constructivisme radical, in L'invention de la réalité. Contribution au constructivisme, sous la dir. de P. Watzlawick, Paris, Seuil, 1988, pp. 19-43.

Glasersfeld Von E, Pourquoi le constructivisme doit être radical ?, Revue des Sciences de l'éducation, 20 (2), pp. 21-29, 1994.

Glasersfeld Von E, Préface, in Désautels J., Larochelle M., Qu'est-ce que le savoir scientifique ? Points de vue d'adolescents et d'adolescentes, Sainte Foy, Presses de l'Université de Laval, 1989.

Goffman E., Frame analysis, New York, Harper and Row, 1974, trad. fr. Les cadres de l'expérience, Paris, éd. de Minuit, 1991.

Goffman I., La situation négligée, in : Les moments et leurs hommes, sous la dir. de Y. Winkin, Paris, Seuil / éd. de Minuit, 1988.

Gonseth F., Le référentiel, univers obligé de médiatisation, Lausanne, L’Age d'Homme, 1975.

Grafmeyer Y. et Joseph I., L'école de Chicago, naissance de l'écologie urbaine, Paris, Aubier, 1990.

Granger G.G., Philosophie, langage, science, Les Ulis, Paris, EDP Sciences, 2003.

Hempel C., Eléments d'épistémologie, Paris, Armand Colin, 1996.

Le Gall Ph., Modélisation économique, in Le dictionnaire des sciences humaines, Paris, PUF, (sous la dir. de S. Mesure et P. Savidan), 2006, pp. 784-786.

Le Moënne C., Quelle conception de la communication interne à l'heure de la dislocation spatiotemporelle des entreprises? in Bulletin interne du groupe Org\&Co, Société française de l'Information et de la communication, octobre 2003.

Le Moënne C., Questions et hypothèses sur les approches constructivistes et les recherches en communications organisationnelles, in La place du constructivisme pour l'étude des communications, actes du colloque de Béziers, CERIC, éd. De l’Université de Montpellier 3, 2004, pp. 9-44.

Le Moigne J.L., Les épistémologies constructivistes, Paris, PUF, 1995.

Le Moigne J.L., La modélisation des systèmes complexes, Paris, Dunod, 1990.

Le Moigne, J.L., La théorie du système général, Paris, PUF, 1984. 
Lessard-Hebert M., Goyette G. et Boutin G., Recherche qualitative : fondements et pratiques, éd. Agence d'Arc inc., Montréal, 1990.

Lesourne J., La notion de système dans les sciences comtemporaines, Colloque CNRS, Lyon, 1980, Librairie de l'Université, Aix-en-Provence, Tome 1 et 2.

Morin E., La méthode, 4 tomes, Paris, Seuil, 1977, 1991.

Morin E., Messie, mais non, conclusion au colloque de Cerisy : Argument pour une méthode, Paris, Seuil, 1990, pp. 254-267.

Morin E., Introduction à la pensée complexe, Paris, éd. ESF, 1990.

Mucchielli A., Place de la systémique des communications dans les diverses systémiques, in Revue internationnale de psychosociologie, éd. Eska, Paris, $\mathrm{n}^{\circ}$ spécial, Psychosociologie et systémique des relations dans les organisations, sous la dir. d'A. Mucchielli et de C. Bourion, 2006, pp. 11-61.

Mucchielli A. et Noy C., Etude des communications : approche constructiviste, Paris, Armand Colin, 2005.

Mucchielli A., Etude des communications : approche par la modélisation des relations, Paris, Armand Colin, 2005.

Mucchielli A., L'interaction problématique-cadrage en systémique des relations, in Questions vives, Etat de la recherche en communication : recherche-action, recherche systémique?, Aix-en Provence Montréal, volume 2, numéro 3, pp. 49-59, septembre 2004.

Mucchielli A., sous la direction de, Le constructivisme dans les études sur la communication, Montpellier, édition de l'Université de Montpellier 3, 314 pages, 2004.

Mucchielli A., L'art d'influencer, Paris, Armand Colin, 2001.

Paillé P. et Mucchielli, A., L'analyse qualitative, Paris, Armand Colin, 2003.

Piaget J., 1937, La construction du réel chez l'enfant, Delachaux-Niestlé, 1980.

Piaget J., La naissance de l'intelligence, Delachaux - Niestlé, 1963.

Pourtois J.P. et Desmet H., Epistémologie et Instrumentation en sciences humaines, Paris, éd. Pierre Mardaga, 1988.

Quéré L., Action située et perception du sens, in La logique des situations. Nouveaux regards sur l'écologie des activités sociales, sous la dir. de M. de Fornel et de L. Quéré, Paris, éd. de l'Ecole des Hautes Etudes en Sciences Sociales, pp. 301-338, 1999.

Quéré L., La situation toujours négligée, in Réseaux, n 85, septembre-octobre 1997, pp. 163-192.

Relieu M., Travaux en public. La dynamique d'une situation problématique, in La logique des situations. Nouveaux regards sur l'écologie des activités sociales, Ouvrage collectif, Paris, éd. de l'Ecole des Hautes Etudes en Sciences Sociales, pp. 995-117, 1999.

Schutz A., Eléments de sociologie phénoménologique, Paris, éd. L’Harmattan, 1998.

Silverman D., La théorie des organisations, Paris, Dunod, 1973.

Sinaceur H., Modèle, in Dictionnaire d'histoire et philosophie des sciences, sous la direction de D. Lecourt), Paris, PUF, 1999, pp. 649-651.

Taylor J. R., La dynamique de changement organisationnel, in Communication \& Organisation, Revue du GREC/O, ISIC, Bordeaux 3, n 3, mai 1993, pp. 50-93. 
Thomas W., Définir la situation, in Y. Grafmeyer et I. Joseph, L'école de Chicago, naissance de l'écologie urbaine, Paris, Aubier, 1990.

Uexkull J. Von, 1956, Théorie de la signification, in Monde des animaux et monde humain, Paris, Denoël, pp. 91-186, 1965.

Varéla F.J., Le cercle créatif. Esquisses pour une histoire naturelle de la circularité, in L'invention de la réalité. Contribution au constructivisme, sous la dir. de P. Watzlawick, Paris, Seuil, 1988, pp. 329-345.

Watzlavick P., Avec quoi consruit-on des réalités idéologiques ?, in :L'invention de la réalité, sous la dir. de P. Watzlawick, Paris,Seuil, 1988, pp. 223-266.

Watzlawick P., L'invention de la réalité, Paris, Seuil, 1980.

Watzlawick P. et al, Une logique de la communication, Paris, Seuil, 1972.

Windish U., Recherche des programmes de vérité, in Dictionnaire des méthodes qualitatives, sous la direction deA. Mucchielli, Paris, Armand Colin, 1995, pp. 172-174.

\section{RÉSUMÉS}

L'article commence par présenter la place des modèles, en général, dans le processus de la connaissance scientifique. Le "modèle " y sera décrit classiquement comme une simplification de la théorie utilisant les principaux concepts de celle-ci pour mieux en montrer les relations. Cette présentation insiste sur l'importance des modèles comme grille a priori d'observation fortement liée aux méthodes utilisées pour appréhender les phénomènes. La présentation examine aussi comment les chercheurs définissent plusieurs types de modèles et plusieurs utilisations de ces modèles dans leurs recherches.

This article first presents the role of models, in general, within the scientific knowledge process. A model is classically described as a simplification of a theory which underlines the relations between the main concepts of this theory. This presentation insists on the importance of models as an observation tool, strongly linked to the methods used to apprehend phenomena. This presentation also examines how researchers defined numerous types of models and numerous used of this models in their studies.

\section{INDEX}

Mots-clés : analyse actionniste, analyse situationnelle, analyse systémique qualitative des communications, contextualisation, contextualisation scientifique, méthode constructiviste, méthode qualitative, modèle, modélisation, situation, système, systémisme

\section{AUTEUR}

\section{ALEX MUCCHIELLI}

Alex Mucchielli est Professeur à l'Université Paul Valéry-Montpellier III, fondateur du département des Sciences de l'Information et de la Communication, responsable du Centre d'Etude et de Recherche en Information et en Communication (CERIC). Mail : alex.mucchielli@gmail.com 